\title{
Identifying Equipment Vendors in Scholarly Publications
}

\author{
Ryan M. Deacon ${ }^{1}$
}

๑) Springer Science+Business Media, LLC, part of Springer Nature and ASM International 2018

When Metallography, Microstructure, and Analysis launched seven years ago, the founding editorial staff decided against having authors specify the vendors of equipment used in their work. The motivation for this decision was to avoid any appearance of partiality or endorsement of any specific instrument manufacturer. The author instructions for $M M A$ state that vendor names are not to be listed in a manuscript, unless there is something unique about a certain vendor's instrumentation.

The editorial staff has recently begun to reconsider the initial decision to not provide instrument vendor names. A basic tenet of scholarly publishing is reproducibility of results-authors need to report sufficient detail of their work so that not only can readers properly interpret the results, but also so that someone practiced in the art could reproduce the work. Furthermore, considering that this journal is devoted to interpreting and analyzing microstructures, there is value in knowing which instrument has been used to prepare and image the microstructures. Identifying the equipment used to produce an image may also help the reviewers and editorial staff in identifying cases of image manipulation.

Although I have not collected accurate numbers, I would estimate that $75 \%$ of the manuscripts submitted to $M M A$ list the instrument vendors in the experimental section. This would seem to indicate that our authors consider this practice standard procedure, likely influenced by their interaction with other journals. A non-exhaustive survey of journals similar to $M M A$ indicates that reporting the equipment vendor would seem to be the norm. Simply thumbing through the journals at my desk (my overflowing "to be read" pile), reveals the following journals permit, if not require, authors to list vendor names in their manuscripts:

Ryan M. Deacon

ryan.deacon@asminternational.org

1 United Technologies Research Center, 411 Silver Lane, East Hartford, CT 06108, USA

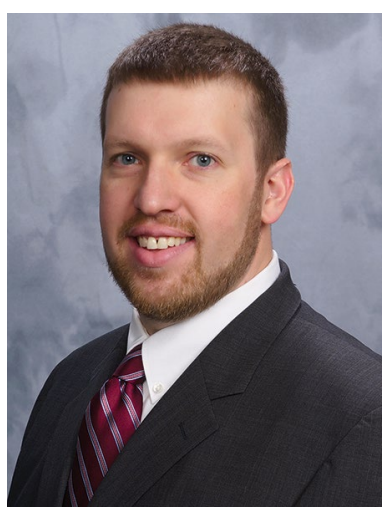

\author{
Journal of Materials \\ Materials Characterization \\ Metallurgical and Materials Transactions \\ Microscopy and Microanalysis \\ Microscopy Today \\ Praktische Metallographie
}

It would seem that $M M A$ is a standout among this group in not permitting the listing of vendor names. I believe that our initial concerns regarding impartiality were overblown. The requirement that our authors be open in reporting their work and fully support efforts at reproducibility far outweighs any concern for implied favoritism.

Starting in 2018, MMA will move to change its current policy, and ask authors to provide vendor names and other pertinent details of the instrumentation used in their work. As this change is enacted, I ask for the input of our readership. If you have thoughts on this decision, I invite you to share them with me directly: ryan.deacon@asminternational.org. 